\title{
Zika Virus Infection: An Emergency of International Concern
}

\author{
Sunny Mandal ${ }^{1}$, Sudhish Mishra ${ }^{2}$, and Prabir K. Mandal ${ }^{3 *}$ \\ ${ }^{1}$ Department of Cell Biology, Microbiology, and Molecular Biology, College of Arts and Sciences, University of South Florida, 4202 East Fowler Avenue, Tampa, \\ FL 33620, USA \\ ${ }^{2}$ Translational Science and Molecular Medicine, Michigan State University, Grand Rapids, MI 48202, USA \\ ${ }^{3}$ Biology Program, Edward Waters College, Jacksonville, FL 32209, USA
}

Received: 13 October, 2016; Accepted: 09 December, 2016; Published: 13 December, 2016

*Corresponding author: Prabir K. Mandal, Biology Program, Edward Waters College, Jacksonville, FL 32209, USA, E-mail: prabir.mandal0807@ewc.edu

\begin{abstract}
The Zika virus (ZIKV) is an arbovirus, meaning that it is insect vector-borne disease, most commonly transmitted through Aedes mosquitoes. ZIKV was first identified in Uganda in 1947 in monkeys through a network that monitored yellow fever. It was later identified in humans. ZIKV is endemic to parts of Africa and Asia, but over the past months, a large number of locally acquired cases have been discovered in Brazil. The number of cases and the locations of the outbreak qualify ZIKV as a rapidly growing epidemic threat. The ZIKV causes microcephaly, small heads and often damaged brains, which may not have developed properly during pregnancy or may have stopped growing in the first years of life. These children may experience a range of problems, like developmental delays, intellectual deficits or hearing loss. Until recently, Zika was not considered a major threat because its symptoms are relatively mild. There is no widely available test for Zika infection. Infection with Zika virus may be suspected based on symptoms and recent history of travel. A diagnosis of Zika virus infection can only be confirmed through laboratory tests on blood or other body fluids, such as urine, saliva or semen. Protection against mosquito bites is a key measure to prevent Zika virus infection.
\end{abstract}

Keywords: Zika virus; Mosquito; Microcephaly; Arbovirus; Centers for disease control and prevention; Dengue; Guillain-Barre syndrome

\section{Introduction}

The ZIKV, a mosquito-transmitted infection related to Dengue, yellow fever and West Nile virus, is an emerging pathogen that has recently been causing serious epidemics around the world. The ZIKV, an arbovirus, and the ZIKV infections spread through infected mosquito bites. "Arbovirus" is a descriptive term applied to hundreds of predominantly RNA viruses that are transmitted by arthropods, notably mosquitoes and ticks. ZIKV is a member of the virus family Flaviviridae and the genus Flavivirus, transmitted by daytime-active Aedes(Stegomyia) mosquitoes, such as Aedesaegypti. Its name comes from the Zika forest of Uganda, where the virus was first isolated in April 1947 from a rhesus macaque monkey that had been placed in a cage near Lake Victoria by the scientists of the Yellow Fever Research Institute [1]. It was subsequently identified in humans in 1952 in Uganda and the United Republic of Tanzania. Cases of ZIKV have previously been reported in Africa, southern Asia, Indonesia, Micronesia, Thailand, the Philippines and the Pacific islands. In 2014, the virus spread eastward across the Pacific Ocean to French Polynesia, then to Easter Island and in 2015 to Mexico, Central America, the Caribbean, and South America [2]. Significant circumstantial evidence links the spread of Aedes mosquitoes during the last summer season in the southern hemisphere with a confluence of environmental factors likely attributable to climate change and the influence of the El Nino effect [3]. This environmental convergence manifests in the form of increased heat and precipitation particularly at lower elevations, which in turn opens up new breeding areas for Aedes mosquitoes. The Pan American Health Organization (PAHO) issued an alert in May 2015 regarding the first confirmed ZIKV infection in Brazil. The outbreak led to reports of a serious neurological condition i.e. Guillain-Barre Syndrome (GBS) as well as pregnant women giving birth to babies with rare and potentially deadly birth defects i.e., microcephaly [4].

ZIKV has a non-segmented, single-stranded, positive-sense RNA genome. Recent preliminary findings from sequences in the public domain uncovered a possible change in nonstructural protein 1 codon usage that may increase the viral replication rate in humans. A mosquito population capable of carrying the ZIKV has been found in a neighborhood of Washington, D.C. and genetic evidence suggests they survived at least the last four winters in the region. Brazil has been labeled the "epicenter" of the ZIKV outbreak, with more than a million cases reported there [5]. In 2016, autochthonous cases of ZIKV infection have been reported from 47 countries. Infection during pregnancy can cause certain birth defects.

\section{Symptoms}

Only female mosquitoes bite people as they need blood in order to lay eggs. They pick up the virus in the blood. It travels from their gut through their circulatory system to their salivary glands and is injected into its next human victim. Mosquito saliva contains proteins that keep blood from clotting. When a mosquito bites it first injects saliva so that its prey's blood does not clog its straw-like proboscis.

The symptoms of ZIKV infection are similar to those of dengue, yellow fever, Japanese encephalitis, West Nile virus and chikungunya diseases spread via the same mosquito that 
transmits ZIKV. The most common symptoms of Zika fever are similar to other arboviruses such as cutaneous maculopapular rash (exanthema), joint pain (arthralgia), low-grade fever, headache, conjunctivitis(bloodshot eyes) and general malaise in about $20 \%$ of the people who contract it, and no symptoms in the other $80 \%$. The muscle pain (myalgia) and headache are not uncommon. ZIKV usually remains in the blood of an infected person for a few days. Severe disease requiring hospitalization is not common and deaths are rare. These symptoms normally last for 2-7 days. Microcephaly is characterized by babies born with incomplete brain development and usually small head sizes, while Guillain-Barre syndrome (which causes the body's immune system to attack its neurological system) can cause paralysis, muscle weakness and other debilitating symptoms in adults.

ZIKV can be passed from a pregnant woman to her fetus. Local mosquito-borne ZIKV transmission has been reported in the continental United States. On February 2, 2016, Dallas County Health and Human Services confirmed the first case of transmission in the United States. According to Centers for Disease Control and Prevention (CDC), Brazilian health authorities reported more than 3,500 microcephaly cases between October 2015 and January 2016.

\section{Diagnosis}

There is no widely available test for Zika infection. Unfortunately, an ultrasound usually cannot detect microcephaly before the end of the second trimester. Some women also should have amniocentesis to test the fluid around the fetus for ZIKV. But amniocentesis involves piercing the amniotic sac with a long needle through the abdomen and it is slightly risky for the fetus and is not recommended before 15 weeks gestation. To detect Zika, a blood or tissue sample from the first week in the infection must be sent to an advanced laboratory so the virus can be detected through sophisticated molecular testing. ZIKV is diagnosed by amplification of viral genome through real time polymerase chain reaction (RT-PCR) [6]. The virus has also been isolated in semen $[7,8]$, and one case of possible person-toperson sexual transmission has been described. There have also been nine people in total who have been diagnosed with the ZIKV in Florida.

\section{Treatment}

No medications or vaccines are available to treat or prevent Zika infections. The suggested treatments recommended by the CDC are very similar to that of like the treatments recommended for dengue. They are to take [1] plenty of rest, [2] drink fluids to prevent dehydration, [3] may take acetaminophen, but do not take aspirin and other non-steroidal anti-inflammatory drugs (NSAIDs) until dengue can be ruled out to reduce the risk of hemorrhage (bleeding). Plans were announced by the authorities in Rio de Janeiro, Brazil to try to prevent the spread of the ZIKV during the 2016 summer Olympic Games in that city. Mosquitoes and their breeding sites pose a significant risk factor for ZIKV infection. The use of Insect repellent, wearing permethrin-coated clothes that cover as much of the body as possible i.e., long sleeves and pants, using physical barriers, closed doors and windows, sleeping under mosquito nets are some of the suggestions made by the World Health Organization (WHO).
Bharat Biotech, a Hyderabad based Indian drug company has claimed that they have developed a vaccine for ZIKV. Two novel approaches that have shown considerable promise in recent years are the genetic control of Aedes aegypti mosquitoes. The first one is known as the Release of insects carrying Dominant Lethal genes (RIDL) and the other strategy is by eliminating Wolbachia which has also been shown to inhibit the replication of additional arboviruses strongly suggesting potential inhibitory effects against ZIKV.

\section{Conclusion}

Zika is prompting worldwide concern because of an alarming connection to a neurological birth disorder and the rapid spread of the virus across the globe. The ZIKV has already spread to 29 countries. The WHO estimates 3 to 4 million people across the Americas will be infected with the virus in the next years. On February 1, 2016, the WHO declared a Public Health Emergency of International Concern (PHEIC) regarding a recent cluster of microcephaly cases and other neurological disorders and the possible association of these illnesses with ZIKV infections. Preventive measures are critical to limit infections transmitted through Aedes aegyptii such as Dengue and Zika virus.

Protection against mosquito bites is a key measure to prevent ZIKV infection. Special attention and help should be given to those who may not be able to protect themselves adequately, such as young children, the sick or elderly. It is important to cover, empty or clean potential mosquito breeding sites in and around houses such as buckets, drums, pots, gutters, and used tires. Research into rapid diagnostics, treatments and vaccines are underway. At present, the only possible intervention for controlling the arboviruses is an extreme reduction in the populations of mosquitoes. The careful reduction of potential breeding places is probably the most important method for their control.

\section{References}

1. World Health Organization. the history of Zika virus. 2016

2. Al-Abdely. An emerging teratogenic virus. Saudi Med J. 2016;37(8):831833. doi:10.15537/smj.2016.8.15676.

3. Cho R. How climate change is exacerbating the spread of disease. 2014. http://blogs.ei.columbia.edu/2014/09/04/how-climate-change-isexacerbating-the-spread-of-disease/

4. Centers for Disease Control and Prevention Symptoms, Diagnosis, \& Treatment. 2016.

5. World Health Organization declares Zika virus public health emergency. 2016.

6. Ventura CV, Maia M, Ventura BV, Linden VVD, Araujo EB, Ramos RC, et al. Ophthalmological findings in infants with microcephaly and presumable intra-uterus Zika virus infection. Arq Bras Oftalmol. 2016;79(1):1-3. doi: 10.5935/0004-2749.20160002.

7. Pan American Health Organization Zika virus infection and Zika fever: Frequently asked questions. 2016.

8. Petersen E, Wilson ME, Touch S, McCloskey B, Mwaba P, Bates M, et al. Unexpected and Rapid Spread of Zika Virus in The AmericasImplications for Public Health Preparedness for Mass Gatherings at the 2016 Brazil Olympic Games. Int J Infect Dis. 2016;44:(11-5). doi: 10.1016/j.ijid.2016.02.001. 